\title{
Extending the scope of the small-ball method
}

\author{
Shahar Mendelson \\ Mathematical Science Institute, \\ The Australian National University, \\ Canberra, Australia \\ and \\ LPSM, Sorbonne University, \\ Paris, France \\ E-mail: shahar.mendelson@anu.edu.au
}

\begin{abstract}
The small-ball method was introduced as a way of obtaining a high probability, isomorphic lower bound on the quadratic empirical process, under weak assumptions on the indexing class. The key assumption was that class members satisfy a uniform small-ball estimate: that $\operatorname{Pr}\left(|f| \geq \kappa\|f\|_{L_{2}}\right) \geq \delta$ for given constants $\kappa$ and $\delta$.

Here we extend the small-ball method and obtain a high probability, almost-isometric (rather than isomorphic) lower bound on the quadratic empirical process. The scope of the result is considerably wider than the small-ball method: there is no need for class members to satisfy a uniform small-ball condition, and moreover, motivated by the notion of tournament learning procedures, the result is stable under a 'majority vote'.
\end{abstract}

\section{Introduction}

In this article we study a more general version of the following question:

Question 1.1. Let $F$ be a class of functions defined on a probability space $(\Omega, \mu)$, let $X$ be distributed according to $\mu$ and consider a sample $X_{1}, \ldots, X_{N}$, consisting of $N$ independent copies of $X$. Find a high probability, lower bound on $\theta=\theta(r)$, defined by

$$
\theta=\inf _{\left\{f \in F:\|f\|_{L_{2}} \geq r\right\}} \frac{1}{N} \sum_{i=1}^{N} \frac{f^{2}\left(X_{i}\right)}{\|f\|_{L_{2}}^{2}},
$$

for a value of $r$ that is as small as possible. 
The obvious implication of (1.1) is if $f \in F$ and $\|f\|_{L_{2}} \geq r$ then

$$
\frac{1}{N} \sum_{i=1}^{N} f^{2}\left(X_{i}\right) \geq \theta\|f\|_{L_{2}}^{2},
$$

which is an 'isomorphic' lower bound on the quadratic empirical process.

Lower bounds on (1.1) play an important role in applications in probability (e.g., the smallest singular value of a random matrix with iid rows), geometry (for example, estimates on the Gelfand widths of convex bodies [14, 15, 1, 13]), and statistics.

The standard way of estimating (1.1) is by two-sided concentration, that is, by obtaining a high probability upper bound on

$$
\sup _{\left\{f \in F:\|f\|_{L_{2}} \geq r\right\}}\left|\frac{1}{N} \sum_{i=1}^{N} \frac{f^{2}\left(X_{i}\right)}{\|f\|_{L_{2}}^{2}}-1\right| .
$$

Estimates of this type are called ratio-limit theorems (see [4, 3] and references therein). However, a nontrivial ratio-limit theorem is possible only if class members have well-behaved tails, and even then obtaining the twosided estimate is rather involved (see, e.g., [13, 12]).

The fact that a high probability, two-sided estimate as in (1.2) is false without assuming that class members have well-behaved tails can be seen by considering what happens for a single function: given a square-integrable function $f$, the probability that

$$
\frac{1}{N} \sum_{i=1}^{N} f^{2}\left(X_{i}\right) \leq C\|f\|_{L_{2}}^{2}
$$

may be small; specifically, it need not be better than the outcome of Chebychev's inequality. Even if one allows for large values of $C$ the situation remains the same: for example, it is straightforward to construct a function $f$ on the unit sphere of $L_{2}(\mu)$ such that

$$
\operatorname{Pr}\left(\frac{1}{N} \sum_{i=1}^{N} f^{2}\left(X_{i}\right) \geq N\right) \geq \frac{c_{1}}{N} .
$$

In contrast, a lower bound of the form

$$
\frac{1}{N} \sum_{i=1}^{N} f^{2}\left(X_{i}\right) \geq c\|f\|_{L_{2}}^{2}
$$

is almost universal and holds with very high probability under minimal assumptions on $f$ : 
Definition 1.2. The function $f$ satisfies a small-ball condition with constants $\kappa>0$ and $0<\delta<1$ if

$$
\operatorname{Pr}\left(|f| \geq \kappa\|f\|_{L_{2}}\right) \geq \delta \text {. }
$$

All that a small-ball condition implies is that $f$ does not assign too much weight to a small neighbourhood of 0 ; it does not mean that $f$ has a well behaved tail, and in particular, it does not exclude the possibility that $f$ does not have any moment beyond the second one. As it happens, a smallball condition is enough to ensure that the lower bound (1.3) holds with very high probability for a well-chosen constant $c$. Indeed, a standard binomial estimate shows that with probability at least $1-2 \exp \left(-c_{1} \delta N\right)$,

$$
\left|\left\{i:\left|f\left(X_{i}\right)\right| \geq \kappa\|f\|_{L_{2}}\right\}\right| \geq \frac{\delta N}{2}
$$

therefore, on that event,

$$
\frac{1}{N} \sum_{i=1}^{N} f^{2}\left(X_{i}\right) \geq \frac{\delta}{2} \kappa^{2}\|f\|_{L_{2}}^{2}
$$

This overwhelming difference between the upper and lower bounds on $\frac{1}{N} \sum_{i=1}^{N} f^{2}\left(X_{i}\right)$ motivated the introduction of the small-ball method [11, 9]. It has led to a lower bound on (1.1) under the assumption that the class in question satisfies a small-ball property - that there are constants $\kappa>0$ and $0<\delta<1$ such that for every $f \in F, \operatorname{Pr}\left(|f| \geq \kappa\|f\|_{L_{2}}\right) \geq \delta$. To formulate this lower bound let

$$
\operatorname{star}(H, f)=\{\lambda h+(1-\lambda) f: h \in H, 0 \leq \lambda \leq 1\}
$$

which is the star-shaped hull of $H$ with $f$. Also, from here on denote by $\left(\varepsilon_{i}\right)_{i=1}^{N}$ independent, symmetric $\{-1,1\}$-valued random variables that are also independent of $\left(X_{i}\right)_{i=1}^{N} ; D$ is the unit ball in $L_{2}(\mu)$; and $S$ is the corresponding unit sphere.

Theorem 1.3. [9] There exist absolute constants $c_{1}$ and $c_{2}$ for which the following holds. Let $H \subset L_{2}(\mu)$ and assume that for every $h \in H, \operatorname{Pr}(|h| \geq$ $\left.\kappa\|h\|_{L_{2}}\right) \geq \delta$. If $r>0$ satisfies that

$$
\mathbb{E} \sup _{h \in \operatorname{star}(H, 0) \cap r S}\left|\frac{1}{\sqrt{N}} \sum_{i=1}^{N} \varepsilon_{i} h\left(X_{i}\right)\right| \leq c_{1} \kappa \delta r \sqrt{N},
$$


then with probability at least $1-2 \exp \left(-c_{2} \delta N\right)$

$$
\inf _{\left\{h \in H:\|h\|_{\left.L_{2} \geq r\right\}}\right.}\left|\left\{i:\left|h\left(X_{i}\right)\right| \geq \frac{\kappa}{2}\|h\|_{L_{2}}\right\}\right| \geq \frac{N \delta}{4} .
$$

In particular, on the same event,

$$
\inf _{\left\{h \in H:\|h\|_{\left.L_{2} \geq r\right\}}\right.} \frac{1}{N} \sum_{i=1}^{N} \frac{h^{2}\left(X_{i}\right)}{\|h\|_{L_{2}}^{2}} \geq \frac{\kappa^{2} \delta}{16} .
$$

Theorem 1.3 has many applications, but our focus here is on three directions in which it is natural to extend its scope:

- Although a small-ball property is a rather minimal condition on a class, there are still important situations in which it is not satisfied. For example, if the class $H$ is a bounded subset of $L_{p}$ for some $p>2$, it need not satisfy a small-ball property. In fact, even if $p=\infty$ and class members are bounded almost surely by 1 , the best possible choice of $\delta$ for a function $h$ may be as bad as $\|h\|_{L_{2}}^{2}$. As an example, fix $0<\rho<1$ and let $h$ be a $\{0,1\}$-valued such that $\operatorname{Pr}(h=1)=\rho$. Therefore, $\|h\|_{L_{2}}^{2}=\rho$ and $\operatorname{Pr}\left(|h| \geq \kappa\|h\|_{L_{2}}\right)=\|h\|_{L_{2}}^{2}$ for any $0<\kappa \leq 1 / \sqrt{\rho}$, and 0 otherwise.

With that in mind, one would like to find a version of Theorem 1.3 that is strong enough to deal with more general situations than classes that satisfy a small-ball property.

- Results that are based on a small-ball property are of an isomorphic nature. The best that one can hope for is that if $h \in H$ and $\|h\|_{L_{2}} \geq r$, then

$$
\frac{1}{N} \sum_{i=1}^{N} h^{2}\left(X_{i}\right) \geq c(\kappa, \delta)\|h\|_{L_{2}}^{2},
$$

where $c(\kappa, \delta)$ depends only on the small-ball parameters $\kappa$ and $\delta$. The fact that $c$ is not close to 1 is unfortunate but unavoidable. On the other hand, at times one requires an almost isometric lower bound, with $c=1-\xi$ for a small $\xi$. Therefore, the second extension of the small-ball method is to ensure that for a fixed $0<\xi<1$ which can be (almost) arbitrarily small, and with high probability one has

$$
\inf _{\left\{h \in H:\|h\|_{L_{2}} \geq r\right\}} \frac{1}{N} \sum_{i=1}^{N} \frac{h^{2}\left(X_{i}\right)}{\|h\|_{L_{2}}^{2}} \geq 1-\xi .
$$


- The final extension is motivated by tournaments [8, 10]. Tournaments are statistical procedures that attain the optimal accuracy/confidence tradeoff for (almost) any prediction problem relative to the squared loss. Roughly and somewhat inaccurately put, consider a class of functions $F$ and an unknown random variable $Y$. One would like to estimate $Y$ by some $f \in F$ whose $L_{2}$ distance to $Y$ is almost the best possible in $F$. The data one is given to perform this task is an iid sample $\left(X_{i}, Y_{i}\right)_{i=1}^{N}$, selected according to the joint distribution of $X$ and $Y$.

To identify which of the class members is almost optimal, one splits the given sample $\left(X_{i}, Y_{i}\right)_{i=1}^{N}$ to $n$ coordinate blocks $\left(I_{j}\right)_{j=1}^{n}$, each one of cardinality $m$; and for any $f, h \in F$ one compares the $n$ empirical errors

$$
\frac{1}{m} \sum_{i \in I_{j}}\left(f\left(X_{i}\right)-Y_{i}\right)^{2} \text { and } \frac{1}{m} \sum_{i \in I_{j}}\left(h\left(X_{i}\right)-Y_{i}\right)^{2} .
$$

Based on the outcomes, one nominates the winner in this "statistical match" between $f$ and $h$, and the key feature of this "tournament" is that if $f$ "wins" in this comparison then $\mathbb{E}(f(X)-Y)^{2}<\mathbb{E}(h(X)-Y)^{2}$. The procedure selects a function that wins all of its matches 1 . As it happens, at the heart of the analysis of tournament procedures is the following question:

Question 1.4. Let $H \subset L_{2}(\mu)$. Fix an integer $n$ and set $\left(I_{j}\right)_{j=1}^{n}$ to be the decomposition of $\{1, \ldots, N\}$ to $n$ blocks of equal size which is denoted by $m$. Given $0<\xi<1$ and $0<\eta<1$, find $r>0$ that is as small as possible such that with high probability, for any $h \in H$ with $\|h\|_{L_{2}} \geq r$

$$
\left|\left\{j: \frac{1}{m} \sum_{i \in I_{j}} h^{2}\left(X_{i}\right) \geq(1-\xi)\|h\|_{L_{2}}^{2}\right\}\right| \geq(1-\eta) n .
$$

Question 1.4 is significantly harder than Question 1.1; for every function in the class whose $L_{2}$ norm is not too small one must show an almost isometric lower bound that holds for a large majority of the coordinate blocks $I_{j}$. Clearly, when $n=1$ and $\eta=0.5$ (or any other constant smaller than 1) and $\xi$ is a constant that need not be small, Question 1.4 reverts to Question 1.1.

\footnotetext{
${ }^{1}$ In actual fact, the choice of a winner of a tournament is more involved. The reason is that when the functions are too close to each other, the outcome of the statistical match between them is unreliable. As a result, the "winner" of the tournament need not be the actual minimizer, but rather a function that is almost as good. For more details, see [8, 10.
} 
Here we answer Question 1.4 without assuming that the class satisfies a small-ball property, thus extending Theorem 1.3 in all the three directions we outlined. The estimate holds, for example, for bounded subsets of $L_{p}$; for classes that satisfies an $L_{q}-L_{2}$ norm equivalence for some $q>2$; and when the class satisfies a uniform integrability condition as in [10].

We end this introduction with some notation. Throughout the article, absolute constants are denoted by $c, c_{1}, \ldots$ and $C, C_{1}, \ldots$. Their value may change from line to line. $c_{p}$ or $c(p)$ means that the constants depend only on the parameter $p$. We write $a \sim b$ when there are absolute constants $c$ and $C$ such that $c a \leq b \leq C a$, and $a \lesssim b$ if only a one-sided inequality holds; $a \sim_{p} b$ and $a \lesssim_{p} b$ implies that the constants depend only on the parameter $p$.

\section{Beyond the small-ball condition}

Before one can extend the small-ball method one must first identify a notion that can replace the small-ball condition. To that end, let us examine the way in which a small-ball condition is used to establish the wanted lower bound.

Given $X_{1}, \ldots, X_{m}$, a small-ball condition with constants $\kappa$ and $\delta$ implies that with very high probability $(1-2 \exp (-c \delta m))$, there are at least $\delta m / 2$ indices $i$ such that $\left|h\left(X_{i}\right)\right| \geq \kappa\|h\|_{L_{2}}$. Thus, not only is

$$
\frac{1}{m} \sum_{i=1}^{m} h^{2}\left(X_{i}\right) \gtrsim_{\kappa, \delta}\|h\|_{L_{2}}^{2},
$$

but (2.1) is stable: discarding a small proportion of the coordinates $\{1, \ldots, m\}$ does not ruin the lower bound.

The notion used in what follows captures these features: not only is $\frac{1}{m} \sum_{i=1}^{m} h^{2}\left(X_{i}\right)$ large enough with high probability, it remains large if any subset of $\{1, \ldots, m\}$ of a reasonable cardinality is discarded from the sum.

Definition 2.1. A function $h$ satisfies a stable lower bound with parameters $(\xi, \ell, k)$ for a sample of cardinality $m$ if with probability at least 1 $2 \exp (-k)$, for any $J \subset\{1, \ldots, m\},|J| \leq \ell$ one has

$$
\frac{1}{m} \sum_{i \in J^{c}} h^{2}\left(X_{i}\right) \geq(1-\xi)\|h\|_{L_{2}}^{2} .
$$

In what follows we do not specify the cardinality of the coordinate block in question (it is denoted by $m$ throughout the article); instead we just say that $h$ satisfies a stable lower bound with parameters $(\xi, \ell, k)$. 


\section{Stability and geometry}

The notion of a stable lower bound has a geometric interpretation. The fact that

$$
\frac{1}{m} \sum_{i=1}^{m} h^{2}\left(X_{i}\right) \geq(1-\xi)\|h\|_{L_{2}}^{2}
$$

obviously means that the random vector $v=\left(h\left(X_{i}\right)\right)_{i=1}^{m}$ is located outside the Euclidean ball $(1-\xi)^{1 / 2} \sqrt{m}\|h\|_{L_{2}} B_{2}^{m}$. Also, with constant probability and in expectation, $\|v\|_{2} \lesssim \sqrt{m}\|h\|_{L_{2}}$, placing $v$ inside a "shell" of inner and outer radius $\sim \sqrt{m}\|h\|_{L_{2}}$. However, all that information says very little about the coordinate distribution of the vector: the fact that $v$ has a Euclidean norm of order $\sqrt{m}\|h\|_{L_{2}}$ does not rule out the possibility that all of its 'mass' is concentrated at a single coordinate. In contrast, a stable lower bound implies that the vector $v$ is well-spread: its $m-\ell$ smallest coordinates still carry significant mass. This fact has a probabilistic implication as well: the (conditional) Bernoulli random variable $\sum_{i=1}^{m} \varepsilon_{i} h\left(X_{i}\right)=\sum_{i=1}^{m} \varepsilon_{i} v_{i}$ exhibits a gaussian-like behaviour. Indeed, it is well known (see [5]) that for every $p \geq 2$ and every $x \in \mathbb{R}^{m}$,

$$
\left\|\sum_{i=1}^{m} \varepsilon_{i} x_{i}\right\|_{L_{p}} \sim \sum_{i \leq p}\left|x_{i}^{*}\right|+\sqrt{p}\left(\sum_{i>p}\left(x_{i}^{*}\right)^{2}\right)^{1 / 2},
$$

where $\left(x_{i}^{*}\right)_{i=1}^{m}$ denotes the nonincreasing rearrangement of $\left(\left|x_{i}\right|\right)_{i=1}^{m}$. If all the mass of $x$ is concentrated at a single coordinate then $\left\|\sum_{i=1}^{m} \varepsilon_{i} x_{i}\right\|_{L_{p}} \sim$ $\|x\|_{2}$, whereas for a gaussian like behaviour one would expect to have that $\left\|\sum_{i=1}^{m} \varepsilon_{i} x_{i}\right\|_{L_{p}}$ is equivalent to $\sqrt{p}\|x\|_{2}$. Thanks to this notion of stability it follows that if $v=\left(h\left(X_{i}\right)\right)_{i=1}^{m}$ then with probability at least $1-2 \exp (-k)$,

$$
\sum_{i \geq \ell}\left(v_{i}^{*}\right)^{2} \geq(1-\xi) m\|h\|_{L_{2}}^{2}
$$

and on that event,

$$
\left\|\sum_{i=1}^{m} \varepsilon_{i} v_{i}\right\|_{L_{p}} \gtrsim \sqrt{p} \sqrt{m}\|h\|_{L_{2}} \sim \sqrt{p \mathbb{E}}\|v\|_{2} \quad \text { for } \quad 2 \leq p \leq \ell .
$$

Remark 2.2. It should be stressed that Definition 2.1 is very different from concentration. If the smaller coordinates of the nonincreasing rearrangement $\left(v_{i}^{*}\right)_{i \geq \ell}$ of a typical realization $v=\left(h\left(X_{i}\right)\right)_{i=1}^{m}$ have 'enough mass' then $h$ satisfies a stable lower bound. However, the larger coordinates $\left(v_{i}^{*}\right)_{i=1}^{\ell}$ can completely destroy any hope of a reasonable upper estimate on $\frac{1}{m} \sum_{i=1}^{m} h^{2}\left(X_{i}\right)$, making two-sided concentration impossible. 


\subsection{Examples of a stable lower bound}

To put the notion of a stable lower bound in some context, let us show that there are many natural situations in which it holds.

\section{A bounded function}

Let $h$ be a function that is bounded almost surely by $M$. As the next lemma shows, $h$ satisfies a stable lower bound.

Lemma 2.3. There are absolute constants $c_{0}$ and $c_{1}$ for which the following holds. Let $h$ be a function that is bounded almost surely by $M$. For any $0<\xi<1$, h satisfies a stable lower bound with parameters $(\xi, \ell, k)$ for

$$
\ell=c_{0} m \xi \frac{\mathbb{E} h^{2}}{M^{2}} \quad \text { and } \quad k=c_{1} m \xi^{2} \frac{\mathbb{E} h^{2}}{M^{2}} .
$$

Proof. Applying Bernstein's inequality, it follows that

$$
\operatorname{Pr}\left(\left|\frac{1}{m} \sum_{i=1}^{m} h^{2}\left(X_{i}\right)-\mathbb{E} h^{2}\right|>u\right) \leq 2 \exp \left(-c m \min \left\{\frac{u^{2}}{\mathbb{E} h^{4}}, \frac{u}{\left\|h^{2}\right\|_{L_{\infty}}}\right\}\right) .
$$

Note that $\mathbb{E} h^{4} \leq M^{2} \mathbb{E} h^{2}$ and $\left\|h^{2}\right\|_{L_{\infty}} \leq M^{2}$. Setting $u=(\xi / 2) \mathbb{E} h^{2}$ it is evident that with probability at least $1-2 \exp \left(-c_{1} m \xi^{2} \mathbb{E} h^{2} / M^{2}\right)$,

$$
\frac{1}{m} \sum_{i=1}^{m} h^{2}\left(x_{i}\right) \geq\left(1-\frac{\xi}{2}\right) \mathbb{E} h^{2}
$$

The contribution to the sum of the $\ell$ largest coordinates is at most $\ell M^{2} / m$, which is at most $(\xi / 2) \mathbb{E} h^{2}$ provided that $\ell \leq m \xi \mathbb{E} h^{2} / 2 M^{2}$, as claimed.

Lemma 2.3 is not very surprising because empirical means of a bounded function exhibit a two-sided concentration around the true mean, which in return implies a stable lower bound. Still, this example is of interest because a bounded function need not satisfy a nontrivial small-ball property.

When leaving the bounded realm the situation is not as straightforward. And the other examples presented here are of that nature: situations in which a stable lower bound holds but there is no hope for a two-sided concentration of the empirical mean.

\section{Tail cutoff}

Because our interest lies in obtaining a lower bound, truncating the function is a possible approach. And, there is a natural location in which the function should be truncated: 
Definition 2.4. For a function $h$ and $0<\xi<1$, set

$$
M(h, \xi)=\inf \left\{t: \mathbb{E} h^{2} \mathbb{1}_{\{|h|>t\}} \leq \frac{\xi}{2} \mathbb{E} h^{2}\right\} .
$$

In other words, $M(h, \xi)$ is the smallest level at which the truncated function $w=h \mathbb{1}_{\{|h| \leq t\}}$ still has a significant $L_{2}$ norm: $\mathbb{E} w^{2} \geq(1-\xi / 2) \mathbb{E} h^{2}$. Applying Lemma 2.3 to the truncated function $h \mathbb{1}_{\{|h| \leq M(h, \xi)\}}$, one has the following:

Corollary 2.5. There are absolute constants $c_{0}$ and $c_{1}$ for which the following holds. If $0<\xi<1$ and $M=M(h, \xi)$ then $h$ satisfies a stable lower bound with parameters $(\xi, \ell, k)$ for

$$
\ell=c_{0} m \xi \frac{\mathbb{E} h^{2}}{M^{2}} \quad \text { and } \quad k=c_{1} m \xi^{2} \frac{\mathbb{E} h^{2}}{M^{2}}
$$

An important example of a tail cutoff, which has been studied in [10] in the context of tournaments, is when one is given a class of functions $H$ such that for any $h \in H, M(h, \xi) \leq \kappa(\xi)\|h\|_{L_{2}}$.

Definition 2.6. A class $H$ satisfies a uniform integrability condition if for every $0<\xi<1$ there is $\kappa(\xi)$ such that for every $h \in H$,

$$
\mathbb{E} h^{2} \mathbb{1}_{\left\{|h| \geq \kappa(\xi)\|h\|_{L_{2}}\right\}} \leq \frac{\xi}{2}\|h\|_{L_{2}}^{2} .
$$

Again, it is standard to verify that each $h \in H$ satisfies a stable lower bound with constants

$$
\ell \sim m \frac{\xi}{\kappa^{2}(\xi)} \quad \text { and } \quad k \sim m \frac{\xi^{2}}{\kappa^{2}(\xi)} .
$$

Once one has more information on $h$, an improved estimate on the cutoff point $M(h, \xi)$ is possible, which also affects the way $\sum_{i=1}^{m} h^{2} \mathbb{1}_{\{|h| \leq M\}}\left(X_{i}\right)$

concentrates around its mean. Two such examples are when $\|h\|_{L_{p}} \leq L$ and when there is norm equivalence between the $L_{q}$ and $L_{2}$ norms, i.e., when $\|h\|_{L_{q}} \leq L\|h\|_{L_{2}}$.

\section{A function bounded in $L_{p}$}

Let $h \in L_{p}$ for some $p>2$. To identify its cutoff point, let $q=p / 2$ and set $q^{\prime}$ to be the conjugate index of $q$. Then

$$
\mathbb{E} h^{2} \mathbb{1}_{\{|h|>t\}} \leq\left(\mathbb{E} h^{2 q}\right)^{1 / q}(\operatorname{Pr}(|h|>t))^{1 / q^{\prime}} \leq\left(\mathbb{E}|h|^{p}\right)^{1 / q} \cdot \frac{\left(\mathbb{E}|h|^{p}\right)^{1 / q^{\prime}}}{t^{p / q^{\prime}}}=\frac{\mathbb{E}|h|^{p}}{t^{p-2}}
$$


Therefore,

$$
M(h, \xi) \leq\left(\frac{2\|h\|_{L_{p}}^{p}}{\xi\|h\|_{L_{2}}^{2}}\right)^{1 /(p-2)}=2^{1 /(p-2)}\|h\|_{L_{p}} \cdot\left(\frac{\|h\|_{L_{p}}^{2}}{\xi\|h\|_{L_{2}}^{2}}\right)^{1 /(p-2)},
$$

and one has

$$
\ell=c_{0} m \xi \frac{\mathbb{E} h^{2}}{M^{2}} \geq c_{1}(p) m\left(\frac{\xi\|h\|_{L_{2}}^{2}}{\|h\|_{L_{p}}^{2}}\right)^{p /(p-2)} .
$$

To identify $k$, set $Z=h^{2} \mathbb{1}_{\{|h| \leq M\}}(X)$, and observe that

$$
\mathbb{E} Z^{2} \leq \begin{cases}c_{2}(p)\|h\|_{L_{p}}^{4}\left(\frac{\|h\|_{L_{p}}^{2}}{\xi\|h\|_{L_{2}}^{2}}\right)^{(4-p) /(p-2)} & \text { if } 2<p<4 \\ \|h\|_{L_{4}}^{4} & \text { if } p \geq 4\end{cases}
$$

Let $Z_{1}, \ldots, Z_{m}$ be independent copies of $Z$. Applying Bernstein's inequality it follows that

$$
\left|\frac{1}{m} \sum_{i=1}^{m} Z_{i}-\mathbb{E} Z\right|>\frac{\xi}{2}\|h\|_{L_{2}}^{2}
$$

with probability at most

$$
\begin{cases}2 \exp \left(-c_{3}(p) m\left(\frac{\xi\|h\|_{L_{2}}^{2}}{\|h\|_{L_{p}}^{2}}\right)^{p /(p-2)}\right) & \text { if } \quad 2<p<4, \\ 2 \exp \left(-c_{4} m \min \left\{\frac{\xi^{2}\|h\|_{L_{2}}^{4}}{\|h\|_{L_{4}}^{4}},\left(\frac{\xi\|h\|_{L_{2}}^{2}}{\|h\|_{L_{p}}^{2}}\right)^{p /(p-2)}\right\}\right) & \text { if } \quad p \geq 4\end{cases}
$$

implying that one may set

$$
k= \begin{cases}c_{3}(p) m\left(\frac{\xi\|h\|_{L_{2}}^{2}}{\|h\|_{L_{p}}^{2}}\right)^{p /(p-2)} & \text { if } 2<p<4, \\ c_{4} m \min \left\{\left(\frac{\xi\|h\|_{L_{2}}^{2}}{\|h\|_{L_{4}}^{2}}\right)^{2},\left(\frac{\xi\|h\|_{L_{2}}^{2}}{\|h\|_{L_{p}}^{2}}\right)^{p /(p-2)}\right\} & \text { if } \quad p \geq 4 .\end{cases}
$$

Remark 2.7. Note that if $p>4$ then $h^{4}=h^{\alpha} h^{4-\alpha}$ for $\alpha=2(p-4) /(p-2)$. By Hölder's inequality for $q=(p-2) /(p-4)$ and $q^{\prime}=(p-2) / 2$, it follows that

$$
\mathbb{E} h^{4} \leq\left(\mathbb{E} h^{2}\right)^{(p-4) /(p-2)} \cdot\left(\mathbb{E}|h|^{p}\right)^{2 /(p-2)}
$$

therefore,

$$
\frac{\|h\|_{L_{2}}^{4}}{\|h\|_{L_{4}}^{4}} \geq\left(\frac{\|h\|_{L_{2}}^{2}}{\|h\|_{L_{p}}^{2}}\right)^{p /(p-2)}
$$

and for $p \geq 4$ one may take

$$
k \sim m \xi^{2}\left(\frac{\|h\|_{L_{2}}^{2}}{\|h\|_{L_{p}}^{2}}\right)^{p /(p-2)}
$$




\section{Norm equivalence}

Another useful example is when $h$ satisfies an $L_{q}-L_{2}$ norm equivalence, i.e, when $\|h\|_{L_{q}} \leq L\|h\|_{L_{2}}$ for some constant $L$. It follows that

$$
M(h, \xi) \leq\left(\frac{2 L^{2}}{\xi}\right)^{1 /(q-2)}\|h\|_{L_{q}}
$$

and one may set

$$
\ell=c_{1}(q) m\left(\frac{\xi}{L^{2}}\right)^{q /(q-2)} \quad \text { and } \quad k= \begin{cases}c_{2}(q) m\left(\frac{\xi}{L^{2}}\right)^{q /(q-2)} & \text { if } \quad 2<q<4 \\ c_{3} m\left(\frac{\xi}{L^{2}}\right)^{2} & \text { if } \quad q \geq 4\end{cases}
$$

\section{The main result}

With the notion of a stable lower bound set in place and armed with the examples, let us formulate the main result of this note. To that end, fix integers $m, n$ such that $N=m n$ and let $\left(I_{j}\right)_{j=1}^{n}$ be the natural partition of $\{1, \ldots, N\}$ to coordinate blocks of cardinality $m$. Recall that $D$ is the unit ball in $L_{2}(\mu)$ and $S$ is the corresponding unit sphere. For $F \subset L_{2}(\mu)$ denote by $\mathcal{M}(F, \rho D)$ the cardinality of a maximal $\rho$-separated subset of $F$ with respect to the $L_{2}(\mu)$ norm.

Theorem 3.1. There exist absolute constants $c_{0}, c_{1}$ and $c_{2}$ for which the following holds. Let $H$ be star-shaped around 0 (i.e., $\operatorname{star}(H, 0)=H$ ) and for $r>0$ set $H_{r}=H \cap r D$. Fix $0<\eta, \xi<1$ and let $r>0$ such that

(1) Every $h \in H \cap r S$ satisfies a stable lower bound with parameters $(\xi / 2, \ell, k)$, for $k \geq \max \{4,2 \log (4 / \eta)\}$.

(2) $\log \mathcal{M}\left(H \cap r S, c_{0} \sqrt{\eta} \xi r D\right) \leq \frac{\eta N}{16} \cdot \frac{k}{m}$.

(3) $\mathbb{E} \sup _{u \in\left(H_{r}-H_{r}\right) \cap c_{0} \sqrt{\eta} \xi r D}\left|\frac{1}{N} \sum_{i=1}^{N} \varepsilon_{i} u\left(X_{i}\right)\right| \leq c_{1} \eta \xi r \cdot \sqrt{\frac{\ell}{m}}$.

Then with probability at least

$$
1-2 \exp \left(-c_{2} \eta N \min \left\{\frac{\ell}{m}, \frac{k}{m}\right\}\right)
$$

we have

$$
\inf _{\left\{h \in H:\|h\|_{L_{2}} \geq r\right\}}\left|\left\{j: \frac{1}{m} \sum_{i \in I_{j}} h^{2}\left(X_{i}\right) \geq(1-\xi)\|h\|_{L_{2}}^{2}\right\}\right| \geq(1-\eta) n .
$$

Moreover, the same assertion holds if one replaces Conditions (2) and (3) with 
(4) $\mathbb{E} \sup _{u \in H_{r}}\left|\frac{1}{N} \sum_{i=1}^{N} \varepsilon_{i} u\left(X_{i}\right)\right| \leq c_{3} \eta \xi r \cdot \min \left\{\sqrt{\frac{\ell}{m}}, \sqrt{\frac{k}{m}}\right\}$, where $c_{3}$ is an absolute constant.

(5) If $h_{1}, h_{2} \in H \cap r S$ and $\left\|h_{1}-h_{2}\right\|_{L_{2}} \geq c_{0} \sqrt{\eta} \xi r$ then $h_{1}-h_{2}$ satisfies a stable lower bound with parameters $(1 / 2, \ell, k)$.

Remark 3.2. In what follows we only consider the more difficult case, in which $0<\xi \leq 1 / 2$, and the required estimate is truly almost isometric rather than isomorphic. We omit the proof of Theorem 3.1 when $1-\xi$ is closer to 0 (e.g., in the situation explored in Theorem 1.3 using the standard small-ball method), which requires a minimal modification of the argument we do present.

Let us mention that Theorem 1.3 is a straightforward outcome of Theorem 3.1 (with slightly different constants) when $H$ satisfies a small-ball property, making Theorem 3.1 a true extension of the small-ball method.

The sufficient condition described in the "moreover" part of Theorem 3.1 can be far from optimal because Condition (4) is significantly more restrictive than the combination of Conditions (2) and (3), forcing one to consider larger values of $r$. Indeed, standard examples of a stable lower bound indicate that often $k \sim \xi \ell$. Therefore, taking the minimum between $\sqrt{\ell / m}$ and $\sqrt{k / m}$ comes at a cost of $\sim \sqrt{\xi}$. Moreover, the indexing set $H \cap r S$ may be much larger than $\left(H_{r}-H_{r}\right) \cap c \sqrt{\xi} r D$. Both factors affect the outcome of Theorem 3.1 when one is looking for a sharp dependence on $\xi$ or when $\xi$ is very small - tending to 0 with $N$. However, when $\xi$ happens to be a fixed constant, the combination of Condition (4) and Condition (5) is a suitable replacement for Conditions (2) and (3).

It is straightforward to apply Theorem 3.1 to any class of functions whose members satisfy a stable lower bound. We chose to focus on one example: a class that is bounded in $L_{p}$ for some $2<p \leq \infty$.

\section{Bounded subsets of $L_{p}$}

The proof of the following corollary is based on the "moreover" part of Theorem 3.1;

Corollary 3.3. Let $p>2$ and assume that $H$ is a bounded class in $L_{p}$, by $M_{p}$. Set $r>0$ such that

$$
\mathbb{E} \sup _{h \in H \cap c_{0} r D}\left|\frac{1}{N} \sum_{i=1}^{N} \varepsilon_{i} u\left(X_{i}\right)\right| \leq c_{1} r\left(\frac{r}{M_{p}}\right)^{p /(p-2)} .
$$


Then with probability at least

$$
1-2 \exp \left(-c_{2} N\left(\frac{r^{2}}{M_{p}^{2}}\right)^{p /(p-2)}\right)
$$

one has

$$
\inf _{\left\{h \in H:\|h\|_{L_{2}} \geq r\right\}}\left|\left\{j: \frac{1}{m} \sum_{i \in I_{j}} h^{2}\left(X_{i}\right) \geq(1-\xi)\|h\|_{L_{2}}^{2}\right\}\right| \geq 0.99 n ;
$$

here $c_{0}$ is a constant that depends only on $\xi$ and $c_{1}$ and $c_{2}$ depend only on $\xi$ and $p$.

Moreover, for $p=\infty$, i.e., if every $h \in H$ satisfies that $\|h\|_{L_{\infty}} \leq M$ and if

$$
\mathbb{E} \sup _{h \in H \cap c_{0} r D}\left|\frac{1}{N} \sum_{i=1}^{N} \varepsilon_{i} u\left(X_{i}\right)\right| \leq c_{1} \frac{r^{2}}{M},
$$

then with probability at least $1-2 \exp \left(-c_{2} N\left(r^{2} / M^{2}\right)\right)$, (3.1) holds; here $c_{0}, c_{1}$ and $c_{2}$ depend only $\xi$.

The case $p=\infty$ can be established using other methods that are based on Talagrand's concentration inequality for empirical processes indexed by bounded subsets in $L_{\infty}$ (see the formulation of Talagrand's theorem in what follows). However, for $p<\infty$ this concentration based argument is no longer valid and as a result estimates like (3.1) where out of reach.

\section{Proof of Theorem 3.1}

The proof of Theorem 1.3, whose general path is followed here as well, is based on three components. Firstly, an individual estimate that holds with high probability - specifically, that with probability at least $1-2 \exp \left(-c_{0} m \delta\right)$,

$$
\frac{1}{m} \sum_{i=1}^{m} h^{2}\left(X_{i}\right) \geq c_{1}(\kappa, \delta)\|h\|_{L_{2}}^{2}
$$

secondly, that this estimate is stable: discarding a reasonable number of coordinates does not significantly affect the sum; and finally, a second type of stability: if $f, h$ are close then the vector $\left((f-h)\left(X_{i}\right)\right)_{i=1}^{N}$ does not have many large coordinates. Once these properties are established, the high probability individual estimate leads to uniform control over a net, and the two notions of stability allow one to pass from the net to the entire class.

The same ideas are used in the proof of Theorem 3.1. Because the claim is homogeneous and $H$ is star-shaped around 0 , it suffices to prove Theorem 
3.1 only for $H \cap r S$. And to deal with $H \cap r S$, one proceeds with the following steps for the pre-determined values of $m$ and $n$ that satisfy $N=m n$ :

(1) For the given choice of $0<\xi<1$, each individual function $h$ satisfies a stable lower bound with parameters $(\xi / 2, \ell, k)$.

(2) Given the $n$ blocks $\left(I_{j}\right)_{j=1}^{n}$ of cardinality $m$, with probability at least $1-2 \exp \left(-c_{0} \eta n k\right)$, the stable lower bound in (1) holds for at least $(1-\eta / 2) n$ blocks.

(3) The high probability estimate in (2) combined with the union bound allows one to obtain (2) for a net in $H \cap r S$, as long as its cardinality is at most $\exp \left(c_{1} \eta n k\right)$ for $c_{1}=c_{0} / 2$.

(4) If $\pi h$ denotes the nearest element to $h$ in the net, stability implies that for at least $(1-\eta / 2) n$ of the blocks, one may discard the set $J_{j}(h)$ consisting of the $\ell$ largest values of the oscillation term $(\mid h-$ $\left.\pi h \mid\left(X_{i}\right)\right)_{i \in I_{j}}$ and still have

$$
\frac{1}{m} \sum_{i \in I_{j} \backslash J_{j}(h)}(\pi h)^{2}\left(X_{i}\right) \geq\left(1-\frac{\xi}{2}\right)\|\pi h\|_{L_{2}}^{2}=\left(1-\frac{\xi}{2}\right) r^{2} .
$$

Hence, for every $h \in H \cap r S$ there are at least $(1-\eta / 2) n$ blocks such that

$$
\begin{aligned}
& \left(\frac{1}{m} \sum_{i=1}^{m} h^{2}\left(X_{i}\right)\right)^{1 / 2} \\
\geq & \left(\frac{1}{m} \sum_{i \in I_{j} \backslash J_{j}(h)}(\pi h)^{2}\left(X_{i}\right)\right)^{1 / 2}-\left(\frac{1}{m} \sum_{i \in I_{j} \backslash J_{j}(h)}(h-\pi h)^{2}\left(X_{i}\right)\right)^{1 / 2} \\
\geq & (1-\xi / 2)^{1 / 2} r-\left(\frac{1}{m} \sum_{i \in I_{j} \backslash J_{j}(h)}(h-\pi h)^{2}\left(X_{i}\right)\right)^{1 / 2} \geq(1-\xi)^{1 / 2} r
\end{aligned}
$$

where the last inequality holds if there is sufficient control on the last term.

Out of this list, (1) is just the stable lower bound; (2) is an immediate outcome of Bennett's inequality; and (3) is the reason for the entropy condition in Theorem 3.1. This leaves us with the crucial point in the proof of Theorem 3.1, which is establishing (4).

To that end, let $\theta_{1}$ be a constant that is specified in what follows, and let $H^{\prime}$ be a maximal $\theta_{1} \xi r$-separated subset of $H \cap r S$. Given a sample $\left(X_{i}\right)_{i=1}^{N}$, let

$$
V=\left\{v=\left((h-\pi h)\left(X_{i}\right)\right)_{i=1}^{N}: h \in H \cap r S\right\}
$$


and put $P_{I_{j}} v=\left(v_{i}\right)_{i \in I_{j}}$. The aim is to ensure that for every $v \in V$ there are at least $(1-\eta / 2) n$ blocks $I_{j}$ such that

$$
\frac{1}{m} \sum_{i>\ell}\left(\left(P_{I_{j}} v\right)_{i}^{*}\right)^{2} \leq \frac{\xi^{2}}{4} r^{2}
$$

implying that for every $h \in H \cap r S$, (4.1) holds for $(1-\eta) n$ blocks.

In other words, if for $h \in H \cap r S$ and $v=\left((h-\pi h)\left(X_{i}\right)\right)_{i=1}^{N}$ one sets

$$
\sharp_{h}=\left|\left\{j: \frac{1}{m} \sum_{i>\ell}\left(\left(P_{I_{j}} v\right)_{i}^{*}\right)^{2}>\frac{\xi^{2}}{4} r^{2}\right\}\right|,
$$

then the main component of the proof of Theorem 3.1 is to show that with high probability,

$$
\sup _{h \in H \cap r S} \sharp_{h} \leq \frac{\eta n}{2} .
$$

Lemma 4.1. There exist absolute constants $c_{1}, c_{2}$ and $c_{3}$ for which the following holds. Let $H$ be star-shaped around 0 , set $\theta_{1}^{2} \leq c_{1} \eta$ and let $H^{\prime}$ to be a maximal $\theta_{1} \xi r$-separated subset of $H \cap r S$ with respect to the $L_{2}(\mu)$ norm. If

$$
\mathbb{E} \sup _{h \in H \cap r S}\left|\frac{1}{N} \sum_{i=1}^{N} \varepsilon_{i}(h-\pi h)\left(X_{i}\right)\right| \leq c_{2} \eta \sqrt{\frac{\ell}{m}} \xi r
$$

then

$$
\operatorname{Pr}\left(\sup _{h \in H \cap r S} \sharp_{h}>\frac{\eta n}{2}\right) \leq 2 \exp \left(-c_{3} N \frac{\ell}{m} \min \left\{\eta, \frac{\eta^{2}}{\theta_{1}^{2}}\right\}\right) .
$$

The proof of Lemma 4.1 is based on Talagrand's concentration inequality for empirical processes indexed by classes of uniformly bounded functions [16], see also [2]:

Theorem 4.2. There exists an absolute constant $C_{0}$ for which the following holds. Let $F$ be a class of functions and set $\sigma_{F}^{2}=\sup _{f \in F} \mathbb{E} f^{2}$ and $b_{F}=$ $\sup _{f \in F}\|f\|_{L_{\infty}}$. Then, for any $x>0$, with probability at least $1-2 \exp (-x)$,

$$
\sup _{f \in F}\left|\frac{1}{N} \sum_{i=1}^{N} f\left(X_{i}\right)-\mathbb{E} f\right| \leq C_{0}\left(\mathbb{E} \sup _{f \in F}\left|\frac{1}{N} \sum_{i=1}^{N} \varepsilon_{i} f\left(X_{i}\right)\right|+\sigma_{F} \sqrt{\frac{x}{N}}+b_{F} \frac{x}{N}\right) .
$$

Proof of Lemma 4.1. Let $A=(m / \ell)^{1 / 2} \xi r$ and set

$$
\phi_{A}(t)=\left\{\begin{array}{lll}
A \cdot \operatorname{sgn}(t) & \text { if } & |t|>A \\
t & \text { if } & |t| \leq A
\end{array}\right.
$$

Given $h \in H \cap r S, \pi h \in H^{\prime}$ and $v_{i}=(h-\pi h)\left(X_{i}\right)$ as above, let

$$
u_{i}=\phi_{A}\left(\left|v_{i}\right|\right) \quad \text { and } \quad w_{i}=\left|v_{i}\right| \mathbb{1}_{\left\{\left|v_{i}\right|>A\right\}}
$$


Note that for every block $I_{j}$,

$$
\min _{J_{j} \subset I_{j},\left|J_{j}\right|=\ell} \frac{1}{m} \sum_{i \in I_{j} \backslash J_{j}}\left(P_{I_{j}} v\right)_{i}^{2} \leq \min _{J_{j} \subset I_{j},\left|J_{j}\right|=\ell} \frac{2}{m} \sum_{i \in I_{j} \backslash J_{j}} u_{i}^{2}+w_{i}^{2},
$$

and if

$$
\frac{1}{m} \sum_{i \geq \ell}\left(P_{I_{j}} v_{i}^{*}\right)^{2}=\min _{J_{j} \subset I_{j},\left|J_{j}\right|=\ell} \frac{1}{m} \sum_{i \in I_{j} \backslash J_{j}}\left(P_{I_{j}} v\right)_{i}^{2} \geq \xi^{2} r^{2} / 4
$$

then either $\frac{1}{m} \sum_{i \in I_{j}} u_{i}^{2} \geq \xi^{2} r^{2} / 16$, or, if the reverse inequality holds, there are at least $\ell$ coordinates in $I_{j}$ such that $w_{i}^{2} \geq A^{2}$. Therefore, if we set

$$
\begin{aligned}
& \sharp_{h}^{1}=\left|\left\{j: \frac{1}{m} \sum_{i \in I_{j}} \phi_{A}^{2}\left(|h-\pi h|\left(X_{i}\right)\right) \geq \frac{\xi^{2} r^{2}}{16}\right\}\right| \text { and } \\
& \sharp_{h}^{2}=\left|\left\{j:\left(|h-\pi h|\left(X_{i}\right)\right)_{\ell}^{*} \geq A\right\}\right|,
\end{aligned}
$$

then

$$
\sharp_{h} \leq \sharp_{h}^{1}+\sharp_{h}^{2} .
$$

Observe that if $\sup _{h \in H \cap r S} \sharp_{h}^{1} \geq \eta n / 4$ then

$$
\sup _{h \in H \cap r S} \sum_{j=1}^{n} \frac{1}{m} \sum_{i \in I_{j}} \phi_{A}^{2}\left(|h-\pi h|\left(X_{i}\right)\right) \geq \frac{\eta n}{4} \cdot \frac{\xi^{2} r^{2}}{16}
$$

that is,

$$
(*)_{1} \equiv \sup _{h \in H \cap r S} \frac{1}{N} \sum_{i=1}^{N} \phi_{A}^{2}\left(|h-\pi h|\left(X_{i}\right)\right) \geq \frac{1}{64} \eta \xi^{2} r^{2} .
$$

Invoking Theorem 4.2, let us show that with high probability, $(*)_{1}<\frac{1}{16} \eta \xi^{2} r^{2}$, and therefore, on that event, $\sup _{h \in H \cap r S} \sharp_{h}^{1}<\eta n / 4$.

Clearly, $\phi_{A}(t) \leq|t|$, and for $\theta_{1}^{2} \leq c_{1} \eta$ and for the right choice of $c_{1}$, it follows that

$$
\mathbb{E} \phi_{A}^{2}\left(|h-\pi h|\left(X_{i}\right)\right) \leq \mathbb{E}|h-\pi h|^{2} \leq \theta_{1}^{2} \xi^{2} r^{2} \leq \frac{1}{256 C_{0}} \eta \xi^{2} r^{2},
$$

where $C_{0}$ is the constant from (4.2).

Also, $\phi_{A}^{2}$ is a Lipschitz function with a constant $2 A$ and satisfies $\phi_{A}^{2}(0)=$ 0 . Thus, by the contraction inequality for Bernoulli processes [7], one can ensure that

$$
\begin{aligned}
\mathbb{E} \sup _{h \in H \cap r S}\left|\frac{1}{N} \sum_{i=1}^{N} \varepsilon_{i} \phi_{A}^{2}\left(|h-\pi h|\left(X_{i}\right)\right)\right| & \leq 2 A \mathbb{E} \sup _{h \in H \cap r S}\left|\frac{1}{N} \sum_{i=1}^{N} \varepsilon_{i}(h-\pi h)\left(X_{i}\right)\right| \\
& \leq \frac{1}{256 C_{0}} \eta \xi^{2} r^{2}
\end{aligned}
$$


provided that

$$
\mathbb{E} \sup _{h \in H \cap r S}\left|\frac{1}{N} \sum_{i=1}^{N} \varepsilon_{i}(h-\pi h)\left(X_{i}\right)\right| \lesssim \eta \xi^{2} \frac{r^{2}}{A} \sim\left(\frac{\ell}{m}\right)^{1 / 2} \eta \xi r
$$

by our choice of $A$.

Turning to the second term, note that for $F=\left\{\phi_{A}^{2}(|h-\pi h|(X)): h \in\right.$ $H \cap r S\}$,

$$
\sigma_{F}^{2} \leq \sup _{h \in H \cap r S} A^{2}\|h-\pi h\|_{L_{2}}^{2} \leq A^{2}\left(\theta_{1} \xi r\right)^{2} \text { and } b_{F} \leq A^{2} .
$$

By Theorem 4.2, $(*)_{1} \leq \frac{1}{16} \eta \xi^{2} r^{2}$ with probability at least

$$
1-2 \exp \left(-c_{3} N \frac{\xi^{2} r^{2}}{A^{2}} \min \left\{\eta, \frac{\eta^{2}}{\theta_{1}^{2}}\right\}\right),
$$

implying that

$$
\operatorname{Pr}\left(\sup _{h \in H \cap r S} \sharp_{h}^{1} \leq \frac{\eta n}{4}\right) \geq 1-2 \exp \left(-c N \frac{\ell}{m} \min \left\{\eta, \frac{\eta^{2}}{\theta_{1}^{2}}\right\}\right) .
$$

Next, note that if $\sharp_{h}^{2} \geq \eta n / 4$, then at least $\ell \eta n / 4$ of the values ( $h-$ $\left.\pi h \mid\left(X_{i}\right)\right)_{i=1}^{N}$ are larger than $A$. To conclude the proof, let us show that with high probability,

$$
\sup _{h \in H \cap r S}\left|\left\{i:|h-\pi h|\left(X_{i}\right) \geq A\right\}\right| \leq \frac{1}{8} \ell \eta n .
$$

Define $\Psi_{A}: \mathbb{R}_{+} \rightarrow \mathbb{R}_{+}$by

$$
\Psi_{A}(t)= \begin{cases}1 & \text { if } \quad t \geq A, \\ \frac{2}{A}\left(t-\frac{A}{2}\right) & \text { if } \quad t \in[A / 2, A), \\ 0 & \text { if } \quad t \in[0, A / 2] .\end{cases}
$$

It is evident that

$$
\sum_{i=1}^{N} \mathbb{1}_{\{|h-\pi h| \geq A\}}\left(X_{i}\right) \leq \sum_{i=1}^{N} \Psi_{A}\left(|h-\pi h|\left(X_{i}\right)\right),
$$

and therefore, it suffices to show that

$$
\sup _{h \in H \cap r S} \frac{1}{N} \sum_{i=1}^{N} \Psi_{A}\left(|h-\pi h|\left(X_{i}\right)\right) \leq \frac{1}{8} \eta \frac{\ell}{m} .
$$

Again, one may invoke Theorem 4.2. Observe that

$$
\mathbb{E} \Psi_{A}\left(|h-\pi h|\left(X_{i}\right)\right) \leq \operatorname{Pr}(|h-\pi h|(X) \geq A / 2) \leq \frac{4\|h-\pi h\|_{L_{2}}^{2}}{A^{2}} \leq \frac{4 \theta_{1}^{2} \xi^{2} r^{2}}{A^{2}} .
$$


Therefore, $\mathbb{E} \Psi_{A}\left(|h-\pi h|\left(X_{i}\right)\right) \leq(\eta / 24) \cdot(\ell / m)$ provided that

$$
\frac{\theta_{1}^{2} \xi^{2} r^{2}}{A^{2}} \lesssim \eta \frac{\ell}{m}
$$

which, by our choice of $A$, holds if $\theta_{1}^{2} \lesssim \eta$.

The function $\Psi_{A}(t)$ is Lipschitz with constant $2 / A$ and $\Psi_{A}(0)=0$. By the contraction inequality for Bernoulli processes,

$$
\begin{aligned}
\mathbb{E} \sup _{h \in H \cap r S}\left|\frac{1}{N} \sum_{i=1}^{N} \varepsilon_{i} \Psi_{A}\left(|h-\pi h|\left(X_{i}\right)\right)\right| & \leq \frac{2}{A} \mathbb{E} \sup _{h \in H \cap r S}\left|\frac{1}{N} \sum_{i=1}^{N} \varepsilon_{i}(h-\pi h)\left(X_{i}\right)\right| \\
& \leq \frac{\eta}{24 C_{0}} \cdot \frac{\ell}{m}
\end{aligned}
$$

as long as

$$
\mathbb{E} \sup _{h \in H \cap r S}\left|\frac{1}{N} \sum_{i=1}^{N} \varepsilon_{i}(h-\pi h)\left(X_{i}\right)\right| \lesssim \eta \frac{\ell}{m} \cdot A \sim \eta \sqrt{\frac{\ell}{m}} \xi r,
$$

and (4.7) follows for our choice of $r$.

Moreover, for every $h \in H \cap r S$,

$$
\mathbb{E} \Psi_{A}^{2}(|h-\pi h|(X)) \leq \operatorname{Pr}(|h-\pi h|(X) \geq A / 2) \leq \frac{4 \theta_{1}^{2} \xi^{2} r^{2}}{A^{2}}
$$

and $\left\|\Psi_{A}(|h-\pi h|)\right\|_{L_{\infty}} \leq 1$. By Theorem 4.2 and recalling once again that $A=(m / \ell)^{1 / 2} \xi r$, one has that

$\operatorname{Pr}\left(\sup _{h \in H \cap r S} \frac{1}{N} \sum_{i=1}^{N} \Psi_{A}\left(|h-\pi h|\left(X_{i}\right)\right) \leq \frac{1}{8} \eta \frac{\ell}{m}\right) \geq 1-2 \exp \left(-c_{6} N \frac{\ell}{m} \min \left\{\eta, \frac{\eta^{2}}{\theta_{1}^{2}}\right\}\right)$,

as claimed.

Thanks to Lemma 4.1, the proof of the first part of Theorem 3.1 follows by showing that there is a net $H^{\prime}$ of $H \cap r S$ whose mesh width is $\theta_{1} \xi r$, and with high probability, each $h^{\prime} \in H^{\prime}$ satisfies an appropriate stable lower bound on at least $(1-\eta / 2) n$ of the blocks.

Lemma 4.3. Let $k \geq \max \{4,2 \log (4 / \eta)\}$ and let $h$ satisfy a stable lower bound with parameters $(\xi / 2, \ell, k)$. Then with probability at least $1-2 \exp (-\eta n k / 8)$ there are at least $(1-\eta / 2) n$ blocks $I_{j}$ such that for any $J_{j} \subset I_{j}$ of cardinality $\ell$,

$$
\frac{1}{m} \sum_{i \in I_{j} \backslash J_{j}} h^{2}\left(X_{i}\right) \geq(1-\xi / 2) \mathbb{E} h^{2} .
$$

Moreover, if $H^{\prime}$ is a class of functions that satisfy such a stable lower bound and $\log \left|H^{\prime}\right| \leq \eta n k / 16$, then with probability at least $1-2 \exp (-\eta n k / 16)$, (4.8) holds for every $h^{\prime} \in H^{\prime}$. 
Proof. Let $\left(\delta_{j}\right)_{j=1}^{n}$ be independent selectors that take the value 0 on the 'good event' one is interested in: that is, each $\delta_{j}$ is a $\{0,1\}$-valued random variable, defined by $\delta_{j}=0$ if for every $J_{j} \subset I_{j}$ of cardinality at most $\ell$ one has

$$
\frac{1}{m} \sum_{i \in I_{j} \backslash J_{j}} h^{2}\left(X_{i}\right) \geq(1-\xi) \mathbb{E} h^{2} .
$$

Therefore, $\mathbb{E} \delta_{j}=\delta \leq 2 \exp (-k)$. If $\eta \geq 4 \exp (-k / 2)$ and $k \geq 4$ then by Bennett's inequality,

$$
\operatorname{Pr}\left(\sum_{j=1}^{n} \delta_{i} \geq \frac{\eta}{2} n\right) \leq \exp \left(-\frac{\eta}{2} n(\log (1+\eta / 2 \delta)-1)\right) \leq \exp (-\eta n k / 8),
$$

as required.

The second part of the claim is evident from the union bound.

Proof of Theorem 3.1, part I. As noted previously, the claim is positive homogeneous, and since $H$ is star-shaped around 0 , it suffices to prove it for $H \cap r S$. For that class, the combination of Lemma 4.3 and Lemma 4.1 leads to the wanted conclusion. Indeed, setting $\theta_{1} \sim \sqrt{\eta}$, by Conditions (1) and (2) there is $H^{\prime} \subset H \cap r S$ that is $\theta_{1} \xi r$-maximal separated and $\log \left|H^{\prime}\right| \leq \eta n k / 16$. Hence, by Lemma 4.3, with probability at least

$$
1-2 \exp (-\eta n k / 4)=1-2 \exp \left(-c_{1} \eta N \frac{k}{m}\right)
$$

for every $u \in H^{\prime}$ there are at least $(1-\eta / 2) n$ blocks $I_{j}$ such that for every $J_{j} \subset I_{j}$ of cardinality at most $\ell$,

$$
\frac{1}{m} \sum_{i \in I_{j} \backslash J_{j}} u^{2}\left(X_{i}\right) \geq(1-\xi / 2)\|u\|_{L_{2}}^{2}=(1-\xi / 2) r^{2} .
$$

Recall that $\pi h$ is the nearest point to $h$ in $H^{\prime}$ relative to the $L_{2}(\mu)$ distance and set $v=\left((h-\pi h)\left(X_{i}\right)\right)_{i=1}^{N}$. By Lemma 4.1, with probability at least

$$
1-2 \exp \left(-c_{2} \eta N \frac{\ell}{m}\right)
$$

for every $h \in H \cap r S$, there are at most $\eta n / 2$ blocks $I_{j}$ such that

$$
\frac{1}{m} \sum_{i>\ell}\left(\left(P_{I_{j}} v\right)_{i}^{*}\right)^{2}>\frac{\xi^{2} r^{2}}{2}
$$

and by (4.1), if (4.9) and (4.10) hold then for every $h \in H \cap r S$ there are at least $(1-\eta) n$ blocks $I_{j}$ such that

$$
\frac{1}{m} \sum_{i \in I_{j}} h^{2}\left(X_{i}\right) \geq(1-\xi) r^{2}=(1-\xi)\|h\|_{L_{2}}^{2} .
$$


Let us turn to the proof of the second part of Theorem 3.1, showing that Conditions (2) and (3) can be replaced by Conditions (4) and (5).

Clearly, if $r$ satisfies Condition (4) for the right choice of constant then it satisfies Condition (3) as well. Therefore, all that is left is to show that Conditions (4) and (5) also imply Condition (2); in particular, that if

$$
\mathbb{E} \sup _{u \in H \cap r D}\left|\frac{1}{N} \sum_{i=1}^{N} \varepsilon_{i} u\left(X_{i}\right)\right| \leq c \eta \sqrt{\xi} r \min \left\{\sqrt{\frac{\ell}{m}}, \sqrt{\frac{k}{m}}\right\},
$$

for the right choice of $c$ and under a stable lower bound, then

$$
\log \mathcal{M}\left(H \cap r S, c_{0} \sqrt{\eta} \xi r D\right) \leq \frac{\eta n k}{16},
$$

where, as always, $\mathcal{M}(F, \rho D)$ is the cardinality of a maximal $\rho$-separated subset of $F$ with respect to the $L_{2}(\mu)$ norm.

Theorem 4.4. There exist absolute constants $c_{0}, c_{1}$ and $c_{2}$ for which the following holds. Let $\rho>0$ and $0<\eta<1$. Assume that for any $h_{1}, h_{2} \in$ $H \cap r S$ that are $\rho$-separated, $h_{1}-h_{2}$ satisfies a stable lower bound with constants $(1 / 2, \ell, k)$ for $k \geq c_{0}$. Assume further that

$$
\log \mathcal{M}(H \cap r S, \rho D) \geq \frac{\eta n k}{16} .
$$

Then with probability at least $1-2 \exp \left(-c_{1} \eta n k\right)$,

$$
\mathbb{E}_{\varepsilon} \sup _{h \in H \cap r S}\left|\frac{1}{N} \sum_{i=1}^{N} \varepsilon_{i} h\left(X_{i}\right)\right| \geq c_{2} \sqrt{\eta} \rho \min \left\{\sqrt{\frac{\ell}{m}}, \sqrt{\frac{k}{m}}\right\} .
$$

Remark 4.5. The constant $1 / 2$ in the stable lower bound may be replaced by any number in $(0,1)$, and that only affects the value of $c_{2}$ in Theorem 4.4.

Applying Theorem 4.4 for the choice of $\rho=c_{0} \sqrt{\eta} \xi r$ shows that Conditions (4) and (5) imply Condition (2). With that, the second part of the theorem follows from the first one.

The proof of Theorem 4.4 is based on Sudakov's inequality for Bernoulli processes [7] in its scale-sensitive formulation (see, e.g., [6]):

Theorem 4.6. There exists an absolute constant $c$ for which the following holds. Let $V \subset \mathbb{R}^{N}$ and for every $v \in V$ set $Z_{v}=\sum_{i=1}^{N} \varepsilon_{i} v_{i}$. If $|V| \geq \exp (p)$ and $\left\{Z_{v}: v \in V\right\}$ is $\varepsilon$-separated in $L_{p}$ then

$$
\mathbb{E} \sup _{v \in V} \sum_{i=1}^{N} \varepsilon_{i} v_{i} \geq c \varepsilon
$$


Proof of Theorem 4.4. Let $h_{1}, h_{2} \in H \cap r S$ such that $\left\|h_{1}-h_{2}\right\|_{L_{2}} \geq$ $\rho$, implying that $h_{1}-h_{2}$ satisfies a stable lower bound with parameters $(1 / 2, \ell, k)$. Hence, by Lemma 4.3, with probability at least $1-2 \exp \left(-c_{1} k n\right)$, there are at least $n / 2$ blocks $I_{j}$ such that for any $J_{j} \subset I_{j}$ of cardinality $\ell$,

$$
\frac{1}{m} \sum_{i \in I_{j} \backslash J_{j}}\left(h_{1}-h_{2}\right)^{2}\left(X_{i}\right) \geq \frac{1}{2} \mathbb{E}\left(h_{1}-h_{2}\right)^{2} .
$$

Without loss of generality assume that the first $n / 2$ blocks are among the 'good blocks', and that their union is $\{1, \ldots, N / 2\}$. Set $v=\left(h_{1}\left(X_{i}\right)\right)_{i=1}^{N}, u=$ $\left(h_{2}\left(X_{i}\right)\right)_{i=1}^{N}$, let $w=v-u$ and consider the random variable

$$
Z_{v}-Z_{u}=\sum_{i=1}^{N} \varepsilon_{i} w_{i}
$$

By a standard contraction inequality [7] and the characterization of the $L_{p}$ norm of the random variable $Z_{a}=\sum_{i=1}^{N} \varepsilon_{i} a_{i}$ from [5], it follows that for $p \geq 1$,

$$
\left\|Z_{v}-Z_{u}\right\|_{L_{p}} \gtrsim\left\|\sum_{i=1}^{N / 2} \varepsilon_{i} w_{i}\right\|_{L_{p}} \sim \sum_{i \leq p} w_{i}^{*}+\sqrt{p}\left(\sum_{i=p+1}^{N / 2}\left(w_{i}^{*}\right)^{2}\right)^{1 / 2}
$$

where $\left(w_{i}^{*}\right)_{i=1}^{N / 2}$ denotes the nonincreasing rearrangement of $\left(\left|w_{i}\right|\right)_{i=1}^{N / 2}$. Set $p=\ell n / 4$ and let $\mathcal{I}$ be the set of indices of the $p$ largest coordinates of $\left(\left|w_{i}\right|\right)_{i=1}^{N / 2}$. Let $J_{j}=\mathcal{I} \cap I_{j}$ and observe that

$$
\left|\left\{j:\left|J_{j}\right| \leq \ell\right\}\right| \geq \frac{n}{4} .
$$

Therefore, by (4.11),

$$
\sum_{i=p+1}^{N / 2}\left(w_{i}^{*}\right)^{2} \geq \frac{n}{4} \cdot \frac{m}{2}\left\|h_{1}-h_{2}\right\|_{L_{2}}^{2} \gtrsim N\left\|h_{1}-h_{2}\right\|_{L_{2}}^{2} .
$$

It follows that for every sample $\left(X_{i}\right)_{i=1}^{N}$ in an event with probability at least $1-2 \exp \left(-c_{1} k n\right)$,

$$
\left\|Z_{v}-Z_{u}\right\|_{L_{p}} \gtrsim \sqrt{p} \cdot \sqrt{N}\left\|h_{1}-h_{2}\right\|_{L_{2}}
$$

and clearly, on the same event, (4.12) holds for any $1 \leq p \leq \ell n / 4$.

Now, let $H^{\prime}$ be a maximal $\rho$-separated subset of $H \cap r S$ and recall that $\log \left|H^{\prime}\right| \gtrsim \eta n k$. By the union bound, with probability at least $1-$ $2 \exp \left(-c_{2} \eta n k\right)$ the random set $V=\left\{\left(h\left(X_{i}\right)\right)_{i=1}^{N}: h \in H^{\prime}\right\}$ contains at 
least $\exp \left(c_{3} \eta n k\right)$ vectors $v$, for which the random variables $\sum_{i=1}^{N} \varepsilon_{i} v_{i}$ are $\sim \sqrt{p} \cdot \sqrt{N} \rho$ separated in $L_{p}$ for any $2 \leq p \leq \ell n / 4$. Set

$$
p \sim n \min \{\eta k, \ell\}=N \min \left\{\frac{\eta k}{m}, \frac{\ell}{m}\right\}
$$

and by Theorem 4.6, with probability at least $1-2 \exp \left(-c_{4} \eta n k\right)$ relative to $X_{1}, \ldots, X_{N}$

$$
\mathbb{E}_{\varepsilon} \sup _{v \in V} Z_{v} \geq c_{5} N \rho \min \left\{\sqrt{\frac{\ell}{m}}, \sqrt{\frac{\eta k}{m}}\right\}
$$

implying that

$$
\mathbb{E}_{\varepsilon} \sup _{h \in H \cap r S}\left|\frac{1}{N} \sum_{i=1}^{N} \varepsilon_{i} h\left(X_{i}\right)\right| \geq c_{5} \sqrt{\eta} \rho \min \left\{\sqrt{\frac{\ell}{m}}, \sqrt{\frac{k}{m}}\right\} .
$$

\section{References}

[1] Shiri Artstein-Avidan, Apostolos Giannopoulos, and Vitali D. Milman. Asymptotic geometric analysis. Part I, volume 202 of Mathematical Surveys and Monographs. American Mathematical Society, Providence, RI, 2015.

[2] S. Boucheron, G. Lugosi, and P. Massart. Concentration inequalities: A Nonasymptotic Theory of Independence. Oxford University Press, 2013.

[3] Evarist Giné and Vladimir Koltchinskii. Concentration inequalities and asymptotic results for ratio type empirical processes. Ann. Probab., 34(3):1143-1216, 2006.

[4] Evarist Giné, Vladimir Koltchinskii, and Jon A. Wellner. Ratio limit theorems for empirical processes. In Stochastic inequalities and applications, volume 56 of Progr. Probab., pages 249-278. Birkhäuser, Basel, 2003.

[5] Paweł Hitczenko. Domination inequality for martingale transforms of a rademacher sequence. Israel J. Math., 84(1-2):161-178, 1993.

[6] Rafał Latała. Sudakov-type minoration for log-concave vectors. Studia Math., 223(3):251-274, 2014.

[7] M. Ledoux and M. Talagrand. Probability in Banach Space. SpringerVerlag, New York, 1991. 
[8] G. Lugosi and S. Mendelson. Risk minimization by median-of-means tournaments. Journal of the European Mathematical Society, To appear.

[9] S. Mendelson. Learning without concentration. Journal of the ACM, 62:21, 2015.

[10] S. Mendelson. An unrestricted learning procedure. Journal of the ACM, 2019.

[11] Shahar Mendelson. A remark on the diameter of random sections of convex bodies. In Geometric aspects of functional analysis, volume 2116 of Lecture Notes in Math., pages 395-404. Springer, Cham, 2014.

[12] Shahar Mendelson. Upper bounds on product and multiplier empirical processes. Stochastic Process. Appl., 126(12):3652-3680, 2016.

[13] Shahar Mendelson, Alain Pajor, and Nicole Tomczak-Jaegermann. Reconstruction and subgaussian operators in asymptotic geometric analysis. Geom. Funct. Anal., 17(4):1248-1282, 2007.

[14] A. Pajor and N. Tomczak-Jaegermann. Nombres de Gelfand et sections euclidiennes de grande dimension. In Séminaire d'Analyse Fonctionelle 1984/1985, volume 26 of Publ. Math. Univ. Paris VII, pages 37-47. Univ. Paris VII, Paris, 1986.

[15] Alain Pajor and Nicole Tomczak-Jaegermann. Subspaces of small codimension of finite-dimensional Banach spaces. Proc. Amer. Math. Soc., 97(4):637-642, 1986.

[16] M. Talagrand. Sharper bounds for Gaussian and empirical processes. Ann. Probab., 22(1):28-76, 1994. 